\title{
Silver nanowire networks as flexible, transparent, conducting films: Extremely high DC to optical conductivity ratios
}

Sukanta De ${ }^{1,2}$, Thomas M Higgins ${ }^{3}$, Philip E Lyons ${ }^{1,2}$, Evelyn M Doherty ${ }^{1,2}$, Peter N. Nirmalraj ${ }^{2,4}$, Werner J Blau ${ }^{1,2}$, John J Boland ${ }^{2,4}$ and Jonathan N Coleman ${ }^{1,2 *}$

${ }^{1}$ School of Physics, Trinity College Dublin, Dublin 2, Ireland

${ }^{2}$ Centre for Research on Adaptive Nanostructures and Nanodevices, Trinity College Dublin, Dublin 2, Ireland

${ }^{3}$ School of Chemistry, University of Wollongong, Wollongong NSW 2522 Australia

${ }^{4}$ School of Chemistry, Trinity College Dublin, Dublin 2, Ireland

We have used aqueous dispersions of silver nanowires to prepare thin, flexible, transparent, conducting films. The nanowires are of length and diameter close to 6.5 $\mu \mathrm{m}$ and $85 \mathrm{~nm}$ respectively. At low thickness, the films consist of networks but appear to become bulk-like for mean film thicknesses above $\sim 160 \mathrm{~nm}$. These films can be very transparent with optical transmittance reaching as high as $92 \%$ for low thickness. The transmittance $(550 \mathrm{~nm})$ decreases with increasing thickness, consistent with an optical conductivity of $6472 \mathrm{~S} / \mathrm{m}$. The films are also very uniform; the transmittance varies spatially by typically $<2 \%$. The sheet resistance decreases with increasing thickness, falling below $1 \Omega /$ for thicknesses above $300 \mathrm{~nm}$. The DC conductivity increases from $2 \times 10^{5} \mathrm{~S} / \mathrm{m}$ for very thin films before saturating at $5 \times 10^{6} \mathrm{~S} / \mathrm{m}$ for thicker films. Similarly, the ratio of DC to optical conductivity increases with increasing thickness from 25 for the thinnest films, saturating at $\sim 500$ for thicknesses above $\sim 160 \mathrm{~nm}$. We believe this is the highest conductivity ratio ever observed for nanostructured films and is matched only by doped metal oxide films. These nanowire films are electromechanically very robust, with all but the thinnest films showing no change in sheet resistance when flexed over $>1000$ cycles. Such results make these films ideal as replacements for indium tin oxide as transparent electrodes. We have prepared films with optical transmittance and sheet resistance of $85 \%$ and $13 \Omega /$ respectively. This is very close to that displayed by commercially available indium tin oxide.

Keywords: Nanowires, electrode, flexible, transparent, conducting.

\footnotetext{
* Corresponding author. Fax: +353 1 6711759. E-mail address: colemaj@tcd.ie (J. Coleman)
} 
Thin, transparent, conducting films are critical for many optoelectronic devices and components. They are most heavily used for electrode applications in devices such as liquid crystal, flat panel or plasma displays, touch panels, organic light-emitting diodes (OLEDs) and solar cells but are also commonly used as antistatic coatings and EMI shielding material. Such films are usually made from doped metal oxides, most commonly indium tin oxide (ITO). However, ITO has a number of drawbacks and is unlikely to be the material of choice in future optoelectronic devices. The difficulties with ITO revolve around the rising cost of indium, the brittleness of ITO and the high temperature processing used in its production. Attempts to resolve the first problem have involved use of alternative metal oxides, ${ }^{1,2}$ thin metal films $s^{3,4}$ or metal grids ${ }^{5,6}$ to prepare transparent conductors. However, the latter two problems are probably more significant; future displays will be larger and will probably reside on a plastic rather than a glass substrate and so must be flexible. Thus, prospective displays will require flexible transparent electrodes that can be produced at low temperature and over large areas at low cost. We note that such requirements are in addition to traditional technical requirements associated with low sheet resistance and high transparency.

It has been known for the last few years that flexibility and low temperature processing can be achieved by deposition of nanostructured thin films from the liquid phase. These are known to be stable under flexing ${ }^{7}$ and can be spray cast ${ }^{8}$, opening the way to large area deposition. While polymer ${ }^{9}$ and graphene ${ }^{10-15}$ films have been studied, the most common material used to date has been carbon nanotubes $^{7,8,16-27}$. While the cost associated with nanotubes is still a factor, it is expected to come down as demand increases to industrial levels. However, the main problem has been achieving transmittance and sheet resistance values routinely achievable with ITO. It is straightforward to achieve sheet resistance of $\sim 10 \Omega /$ for transmittance of $>90 \%$ with ITO. Such values have not been achieved with nanostructured electrodes. A commonly used figure of merit for transparent conductors is the ratio of DC to optical conductivity, $\sigma_{D C} / \sigma_{O p}$. To achieve $R_{s}=10 \Omega /$ and $T=90 \%$ requires $\sigma_{\mathrm{DC}} / \sigma_{\mathrm{Op}}=350$ (see below). The best results for graphene based films ${ }^{14}$ have been $\sigma_{\mathrm{DC}} / \sigma_{\mathrm{Op}}=0.5$ while for nanotubes ${ }^{20} \sigma_{\mathrm{DC}} / \sigma_{\mathrm{OP}}=25$ has been demonstrated. We propose that this gap can be bridged by preparing thin films of metallic nanowires. Lee et al. have pioneered this method by preparing networks of silver nanowires (AgNWs) for which we calculate $\sigma_{\mathrm{DC}} / \sigma_{\mathrm{Op}} \sim 150$. In this paper we demonstrate a method to produce thin films of silver nanowires with $\sigma_{\mathrm{DC}} / \sigma_{\mathrm{Op}}=500$, similar to that required to match ITO. These films have DC conductivity of up to $5 \times 10^{6} \mathrm{~S} / \mathrm{m}, 8 \%$ of the value for bulk silver. We have prepared films with $\mathrm{R}_{\mathrm{s}}=13 \Omega /$ coupled with $\mathrm{T}=85 \%$. These films are stable under flexing for at least 1000 cycles.

Results and Discussion 
Shown in figure 1A is a photograph of a nanowire film on a PET substrate. This was prepared by depositing $28 \mathrm{mg} / \mathrm{m}^{2}$ of AgNWs onto a cellulose membrane by vacuum filtration. The film was then attached to the PET substrate using heat and pressure (see methods) and the cellulose dissolved in acetone. It is clear from this image that the film appears to be of very high optical quality. While this is generally true, we note that the films appear very slightly milky due to light scattering from the nanowires $^{28}$. We can test the optical uniformity of these films by taking a transmission scan of the deposited film. This involves measurement of the local white light transmittance with a spatial resolution of $4 \mu \mathrm{m}$, measured over an area of $2 \mathrm{~mm} \times 2 \mathrm{~mm}$. We can characterise the uniformity of the transmittance by calculating the standard deviation of the transmittance over all pixels, $\Delta \mathrm{T}$. This worked out to be $\Delta \mathrm{T}=1.8 \%$ for the film shown in figure $1 \mathrm{~A}$, demonstrating the optical quality of these films. When normalised to transmittance, this gives $\Delta T /\langle T\rangle=2.1 \%$. We will use this technique below to investigate the film uniformity as a function of film thickness.

We have prepared a range of films with varying thickness by filtering different dispersion volumes to give different deposited masses. We characterise these by their deposited mass per unit area, M/A. We investigate the nature of these films using scanning electron microscopy as shown in figure 1 B-E. To facilitate SEM analysis we transferred these films onto Au/Pd coated glass after filtration. Shown in figure $1 B$ is an SEM image for an $M / A=46 \mathrm{mg} / \mathrm{m}^{2}$ film. This image shows a network of nanowires that, while clearly above percolation, can be considered sparse. As a result, there is significant non-uniformity, with holes in the network (where the substrate is visible) of sizes ranging from 2-6 $\mu \mathrm{m}$ in diameter. In figure $1 \mathrm{C}-\mathrm{E}$ we show successive images for films of increasing thickness (M/A increasing from $93-780 \mathrm{mg} / \mathrm{m}^{2}$ ). It is clear from these images that, as the thickness is increased, the networks become less sparse with the substrate appearing less frequently. In addition, the films appear more uniform; by $780 \mathrm{mg} / \mathrm{m}^{2}$, the substrate cannot be seen at all and the network appears spatially homogenous. From images such as the one shown in figure 1B, we can measure the length and diameter of the nanowires. This data is shown in figure $1 \mathrm{~F}$ and $\mathrm{G}$ in the form of histograms. The length varies from $2.5-15 \mu \mathrm{m}$ with a mean of $6.6 \mu \mathrm{m}$. The diameter varies from $50-150 \mathrm{~nm}$ with a mean of $84 \mathrm{~nm}$. We note that these lengths are larger than those found for most nanotubes while the diameters are almost an order of magnitude larger than that usually found for nanotube bundles in thin films.

We have measured the transmittance spectra in the visible region for all films studied as shown in figure 2A. The spectra were reasonably featureless although those representing very thin films displayed a number weak, broad peaks. From these spectra, we measured the transmittance at $550 \mathrm{~nm}$, $\mathrm{T}$, and sheet resistance, $\mathrm{R}_{\mathrm{s}}$, for all the films studied in this work as shown in figure $2 \mathrm{~B}$. We find that for the thinnest films $\left(\mathrm{M} / \mathrm{A}=28 \mathrm{mg} / \mathrm{m}^{2}\right.$ ), $\mathrm{T}$ approaches $92 \%$ for sheet resistance approaching $100 \Omega /$. 
For thick films $\left(\mathrm{M} / \mathrm{A}=230 \mathrm{mg} / \mathrm{m}^{2}\right)$, $\mathrm{T}$ approaches $32 \%$ for $\mathrm{R}_{\mathrm{s}} \sim 0.5 \Omega /$. In the middle range, which is of most interest for electrode applications, the film with $\mathrm{M} / \mathrm{A}=70 \mathrm{mg} / \mathrm{m}^{2}$ displays $\mathrm{T}=75 \%$ and $\mathrm{R}_{\mathrm{s}}=3.4$ $\Omega /$. In general, the transmittance and sheet resistance for thin metallic films are related by: ${ }^{29}$

$$
T(\lambda)=\left(1+\frac{188.5}{R_{s}} \frac{\sigma_{O p}(\lambda)}{\sigma_{D C}}\right)^{-2} \text { Eq } 1
$$

where $\sigma_{\mathrm{Op}}(\lambda)$ is the optical conductivity (here quoted at $\lambda=550 \mathrm{~nm}$ ) and $\sigma_{\mathrm{DC}}$ is the DC conductivity of the film. This expression has previously been shown to accurately describe films of both carbon nanotubes $^{20}$ and polymer-nanotube composites ${ }^{7}$. This expression has been fitted to the data in figure $2 \mathrm{~B}$ and provides a reasonable fit for $\mathrm{M} / \mathrm{A}>70 \mathrm{mg} / \mathrm{m}^{2}$. It is clear from figure $1 \mathrm{~B}$ that films with $\mathrm{M} / \mathrm{A}<70$ $\mathrm{mg} / \mathrm{m}^{2}$ are quite sparse and perhaps should be considered as $2 \mathrm{D}$ networks rather than $3 \mathrm{D}$ films. With this in mind we can imagine a network to film transition occurring at $\mathrm{M} / \mathrm{A} \sim 70 \mathrm{mg} / \mathrm{m}^{2}$. The fit to equation 1 shown in figure $2 \mathrm{~B}$ is described completely by $\sigma_{\mathrm{DC}} / \sigma_{\mathrm{OP}}=500$. This ratio can be considered as a figure of merit for thin conducting films. A value of $\sigma_{\mathrm{DC}} / \sigma_{\mathrm{Op}}=500$ is extremely large for a nanostructured thin film. Previous work has demonstrated a maximum value of by $\sigma_{\mathrm{DC}} / \sigma_{\mathrm{Op}}=25$ for acid treated films of SWNTs. ${ }^{20}$ We can illustrate this by plotting on figure $2 \mathrm{~B}$ data measured in our lab for near state-of-the-art nanotube films (Iljin Nanotech, no post-treatment ${ }^{30}$ ). This data is described by $\sigma_{\mathrm{DC}} / \sigma_{\mathrm{Op}}=13$, significantly worse than that displayed by the AgNW films. We note that this transmittance - sheet resistance data is superior to all data we have found in the literature for nanostructured thin films. In addition, the values presented in figure $2 \mathrm{~B}$ are slightly better then data recently reported for metallic grids. ${ }^{5}$

To put this data in context, we prepared thin silver films with thickness 25,35 and $50 \mathrm{~nm}$ by evaporation (thinner films oxidised rapidly rendering them quite resistive). We measured the transmittance (see figure $2 \mathrm{~A}$ ) and sheet resistance to vary in the ranges $10 \%<\mathrm{T}<40 \%$ and $0.7 \Omega /<\mathrm{R}_{\mathrm{s}}<1.8 \Omega /$ as shown in figure 3 . This data is well described by equation 1 with $\sigma_{\mathrm{DC}} / \sigma_{\mathrm{OP}}=143$, far below that measured for AgNW films. In addition we measure $\mathrm{T}$ and $\mathrm{R}_{\mathrm{s}}$ for two films of commercially available ITO. These films had $\mathrm{T} \sim 97 \%$ and $\mathrm{R}_{\mathrm{s}} \sim 15-30 \Omega /$, giving $\sigma_{\mathrm{DC}} / \sigma_{\mathrm{OP}}=400-800$, slightly better that measured for our AgNW films. It is clear from this graph that our AgNW films are significantly better than solid silver films but slightly inferior to ITO. We will discuss this in more detail below.

In order to measure bulk film properties such as by $\sigma_{\mathrm{Op}}$ and $\sigma_{\mathrm{DC}}$, we need to relate $\mathrm{M} / \mathrm{A}$ to the film thickness, $t$. To do this we prepared a relatively thick film with $\mathrm{M} / \mathrm{A}=780 \mathrm{mg} / \mathrm{m}^{2}$. This film was thick enough to measure its thickness by SEM giving $\mathrm{t}=1800 \mathrm{~nm}$. Knowing the thickness and mass per unit area allows us to calculate the film density from: $M / A=\rho t$, giving $\rho=435 \mathrm{~kg} / \mathrm{m}^{3}$. This allows us to calculate the porosity of this film to be $96 \%$. Assuming the film density is invariant with M/A (an 
assumption that is probably less valid at lower thickness), we can calculate an average film thickness for all films. We note that such thickness values should be treated with scepticism for $\mathrm{M} / \mathrm{A}<70 \mathrm{mg} / \mathrm{m}^{2}$ $(\sim 160 \mathrm{~nm})$ below which the films should be considered 2D networks.

Shown in figure $3 \mathrm{~A}$ is a graph of transmittance $(550 \mathrm{~nm})$ as a function of film thickness. For thin metallic films, ${ }^{29}$ the transmittance, $\mathrm{T}$, scales with thickness, $\mathrm{t}$, as

$$
T(\lambda)=\left(1+188.5 \sigma_{O p}(\lambda) t\right)^{-2} \quad \operatorname{Eq} 2
$$

That equation 2 is appropriate for these networks is emphasised by the inset, which shows that the data is fitted extremely well by this model when appropriately linearised. This equation has been fit to the data in figure $3 \mathrm{~A}$ over its whole range, yielding a value of $\sigma_{\mathrm{Op}}=6472 \mathrm{~S} / \mathrm{m}$. This value is surprisingly low and compares with $\sigma_{\mathrm{Op}}=1.75 \times 10^{5} \mathrm{~S} / \mathrm{m}$ as measured for our thin evaporated silver films. This is lower than the reported value of $9.4 \times 10^{6} \mathrm{~S} / \mathrm{m}$ for bulk silver. ${ }^{31}$ In any case, it is unclear why these AgNW networks should display such low values of $\sigma_{\mathrm{Op}}$. The measured value is just $0.07 \%$ of the value for bulk silver, a factor that cannot be explained solely by the high film porosity. In addition, the optical conductivity is lower than values measured for thin nanotube films $\left(1.5-2 \times 10^{4} \mathrm{~S} / \mathrm{m}, \lambda=550\right.$ $\mathrm{nm}) .^{20,30,32}$

Shown in figure $3 \mathrm{~B}$ is the sheet resistance data as a function of film thickness. It can be seen that this data does not scale inversely with thickness as would be expected for a bulk material (illustrated by the dashed line). This can be seen more clearly by plotting the data as DC conductivity versus thickness using $\sigma_{D C}=1 /\left(R_{s} t\right)$ as shown in figure $3 \mathrm{C}$. The DC conductivity is not thickness invariant but increases with increasing thickness from $\sim 2 \times 10^{5} \mathrm{~S} / \mathrm{m}$ for the $60 \mathrm{~nm}$ thick film up to $\sim 5 \times 10^{6} \mathrm{~S} / \mathrm{m}$ for the $600 \mathrm{~nm}$ thick film. The conductivity appears to approach saturation for thicknesses greater than $160 \mathrm{~nm}\left(\mathrm{M} / \mathrm{A}<70 \mathrm{mg} / \mathrm{m}^{2}\right)$ i.e. above the network to bulk transition. We note that these conductivities are extremely high with the maximum values approaching $8 \%$ of bulk silver $\left(6.3 \times 10^{7}\right.$ $\mathrm{S} / \mathrm{m})$. We note that this fraction $(8 \%)$ is close to the fractional volume of film filled by nanowires $(4 \%)$. It is also worth noting that we measured the conductivity of our evaporated silver films to be $2.6 \times 10^{7} \mathrm{~S} / \mathrm{m}$. We attribute the difference between this value and the bulk value to the presence of a thin oxide layer. These AgNW films also display conductivities much higher than the highest conductivity observed for a nanotube films; $\sim 6 \times 10^{5} \mathrm{~S} / \mathrm{m} .{ }^{25}$ As the conductivity of nanostructured films such as these is limited by the presence of inter-wire bundles, ${ }^{33}$ we suggest that the effective junction resistance in these $\mathrm{AgNW}$ films is exceptionally low. Previously, ${ }^{28}$ computational studies have suggested junction resistances of $\sim 1 \Omega$. This is extremely low compared with estimates of nanotubenanotube junction resistances of $\sim 50 \mathrm{k} \Omega .^{34}$

We can also use equation 1 to calculate the conductivity ratio, $\sigma_{\mathrm{DC}} / \sigma_{\mathrm{Op}}$, as a function of film thickness as shown in figure 3D. Unsurprisingly, this data resembles the DC conductivity data with 
$\sigma_{\mathrm{DC}} / \sigma_{\mathrm{Op}}$ increasing with increasing thickness from $\sim 25$ for the $60 \mathrm{~nm}$ thick film up to $\sim 500$ for the 600 $\mathrm{nm}$ thick film. In addition, like the DC conductivity, $\sigma_{\mathrm{DC}} / \sigma_{\mathrm{Op}}$ begins to saturate above the network to bulk transition. These conductivity ratios are exceptionally high compared with other systems. Bulk silver $^{31}$ has been reported to show $\sigma_{\mathrm{DC}} / \sigma_{\mathrm{Op}} \sim 7$ although we measured $\sigma_{\mathrm{DC}} / \sigma_{\mathrm{Op}}=143$ for the evaporated thin silver films. In comparison, the highest value recorded for nanotube films ${ }^{20}$ has been $\sigma_{\mathrm{DC}} / \sigma_{\mathrm{Op}} \sim 25$. Only doped metal oxides such as have comparable values $\left(\sigma_{\mathrm{DC}} / \sigma_{\mathrm{Op}} \sim 400-800\right)$.

As mentioned above, the DC conductivity and conductivity ratio tend to fall off for thicknesses below the network to film transition. We can explore this transition in more detail by measuring the local non-uniformity of the films as a function of thickness. We do this by recording transmission scans (pixel size $4 \mu \mathrm{m}$ ) of a number of the films discussed above. We transform the resulting transmission maps into absorbance (A) maps using $A=-\log T$. By the Lambert Beer law, the absorbance is proportional to the number of absorbing objects per unit area. This means the absorbance map is a measure of the spatial distribution of nanowires per $4 \mu \mathrm{m}$ pixel. For our purposes, we define the non-uniformity as the standard deviation of absorbance as measured over a $2 \mathrm{~mm} \times 2 \mathrm{~mm}$ grid (500×500 pixels). We associate a large degree of non-uniformity with a high standard deviation. We plot the data for non-uniformity as a function of film thickness in figure $3 \mathrm{E}$. It is clear that the nonuniformity is reasonably constant for thicker films but increases significantly for film with thickness below $150 \mathrm{~nm}$. This is very close to the estimated network to film transition thickness of $160 \mathrm{~nm}$ $\left(\mathrm{M} / \mathrm{A}<70 \mathrm{mg} / \mathrm{m}^{2}\right)$. In fact figures $3 \mathrm{~B}-\mathrm{E}$ are all similar in that in all cases the data shown deviates strongly from the high thickness value for thicknesses below $\sim 150-170 \mathrm{~nm}$, emphasising the presence of a network to film transition.

An additional condition for a thin film to act as an electrode is that current can effectively flow out of the plane of the film as well as through it. To test this we performed conductive AFM (C-AFM). Shown in figure $4 \mathrm{~A}$ is an AFM topographical image of the surface of a sparse AgNW network $\left(\mathrm{M} / \mathrm{A}=10 \mathrm{mg} / \mathrm{m}^{2}\right)$. All AFM images recorded show a network of straight, well defined nanowires, lying in the plane of the film. While the vast majority of wires are connected to the network, a small number are isolated. Shown in figure 4B is a conductive-AFM current map of the same area of the network shown in figure 4A. The current map is very similar to the topographical map and clearly shows a nanowire network. This map unambiguously shows that current can flow out of the plane of the film from the wire sidewalls to the AFM tip. This is important as it shows that current can be gathered uniformly from all areas of the surface of these films - a critical property for any material with potential for use as an electrode. We note that none of the nanowires in the network are orientated out of the plane of the film. The current is collected from the sidewall of the wires. 
As mentioned above, these films have optical and electrical properties close to those measured for ITO. However, to surpass ITO as an electrode these films need to remain transparent and conducting under flexing. Such a material is of considerable interest as an electrode in applications such as e-paper. To test this, we prepared AgNW films on PET at a number of thicknesses. In each case we monitored the sheet resistance during bending with the AgNW film in compression and in one case in tension. The films were bent from an initial radius of curvature of $7.5 \mathrm{~mm}$ to a final radius of $2.5 \mathrm{~mm}$ before being relaxed. This was repeated over many bend cycles. Shown in figure 5A-C is the mean sheet resistance per cycle plotted versus cycle number for three AgNW films of different thickness. The thinnest film $\left(\mathrm{M} / \mathrm{A}=39 \mathrm{mg} / \mathrm{m}^{2}, \mathrm{t}=90 \mathrm{~nm}\right)$ showed reasonably stable sheet resistance over the first 200 cycles. However, at this point the film began to fail, with the sheet resistance increasing by two orders of magnitude over the next 2000 cycles. In contrast, the sheet resistance of the thicker films was virtually unchanged over $\sim 2000$ cycles with a deviation of $<2 \%$ around the mean. For the $\mathrm{M} / \mathrm{A}=79 \mathrm{mg} / \mathrm{m}^{2}$ film $(\mathrm{t}=180 \mathrm{~nm}$ ), we measured the sheet resistance for both compressive and tensile bending as shown in figure $5 \mathrm{C}$. Both data sets showed little variation in resistance although the results of the tensile test were slightly noisier. For comparison we measured the electromechanical stability of a solid silver film with thickness $25 \mathrm{~nm}$ as shown in figure 5D. Here the sheet resistance was as stable as the thicker AgNW films. This is in stark contrast to thin ITO films which have been reported to fail catastrophically after only 160 bend cycles. $^{7}$ Note that none of the films studied in this work failed during these measurements. In each case, the number of cycles was limited by time constraints.

However, one problem with these materials is their adhesion to PET. We performed scotch tape tests on AgNW films of thicknesses of $150 \mathrm{~nm}$ and $430 \mathrm{~nm}$ on PET. Unlike carbon nanotube films, both AgNW films failed the test, being completely removed from the substrate. While this is a problem, we don't feel it is a fatal one. It is likely that PET can be chemically treated to improve the adhesion. We feel this is a technical problem that will be solved with further study.

We note that above the network to bulk transition, these AgNW films display a figure of merit $\left(\sigma_{\mathrm{DC}} / \sigma_{\mathrm{Op}}\right)$ of $\sim 500$, slightly lower than that measured for ITO $\left(\sigma_{\mathrm{DC}} / \sigma_{\mathrm{Op}} \sim 400-800\right)$. However, unlike ITO, ${ }^{7,35,36}$ these films are electromechanically robust under flexing. However, we currently cannot simultaneously achieve transmittance and sheet resistance to match ITO. To reach transmittance greater than $90 \%$ we need to prepare reasonably thin films. Unfortunately, such films have thicknesses below the network to film transition and as such have reduced values of $\sigma_{\mathrm{DC}} / \sigma_{\mathrm{Op}}$ and so reduced $\sigma_{\mathrm{DC}}$. The best results we could achieve were $R_{s}=13 \Omega /$ for $T=85 \%\left(M / A=47 \mathrm{mg} / \mathrm{m}^{2}, t=107 \mathrm{~nm}\right)$. This film is below the network to film transition and as such displays significant non-uniformity as illustrated in figure 3E. This non-uniformity will pose a significant problem for real applications. We propose that these issues could be resolved simply by forcing the network to film transition to occur at lower thicknesses. This could be done simply by using AgNWs with lower diameter as the transition 
thickness is probably defined by some multiple of the wire diameter. We note that a similar transition has been observed in films of single wall nanotube bundles (diameter $\sim 20 \mathrm{~nm}$ ) to occur at $\mathrm{t}=40 \mathrm{~nm}^{30}$ In our work we observe the transition at $\mathrm{t} \sim 160 \mathrm{~nm}$ for wires with $\mathrm{D}=84 \mathrm{~nm}$. In both cases, the transition occurs for film thicknesses of approximately twice the nanowire diameter. In order to achieve $\mathrm{T}>90 \%$ coupled with $\sigma_{\mathrm{DC}} / \sigma_{\mathrm{OP}}>400$, we need to shift the network to film transition to thicknesses below 50nm (see fig 3). If the transition occurs for thicknesses of twice the wire diameter, this means we need wires with diameters below $25 \mathrm{~nm}$. We note that, in addition to improving T, using such low diameter wires should have an additional effect. It has recently been shown that the conductivity of networks of nanowires increases as the wire diameter decreases. ${ }^{33,37}$ This means that using lower diameter nanowires may result in further increases in $\sigma_{\mathrm{DC}} / \sigma_{\mathrm{OP}}$, as well as reduction in the transition thickness. We note that recently gold nanowires have been reported with diameters as low as $6 \mathrm{~nm} .{ }^{38}$ We believe that using such wires should result in high work-function, spatially uniform, flexible electrodes with high transmittance and extremely high DC conductivity.

In conclusion, we have prepared thin transparent, conductive films from silver nanowires. For thicknesses below $\sim 160 \mathrm{~nm}$, these films act like a 2D network while thicker films are more bulk-like. The optical transmittance decreases with increasing thickness as described by an optical conductivity of $\sigma_{\mathrm{Op}}=6472 \mathrm{~S} / \mathrm{m}(550 \mathrm{~nm})$. The DC conductivity increases with thickness from $\sim 2 \times 10^{5} \mathrm{~S} / \mathrm{m}$ for low thicknesses, saturating close to $5 \times 10^{6} \mathrm{~S} / \mathrm{m}, 8 \%$ of the bulk silver value. This results in films with sheet resistance $<10 \Omega /$ as long as the thickness is $>100 \mathrm{~nm}$. In addition the ratio, $\sigma_{\mathrm{DC}} / \sigma_{\mathrm{Op}}$ increases with increasing thickness, saturating close to 500 for thicknesses $>200 \mathrm{~nm}$. Our best film had $R_{s}=13 \Omega /$ for $\mathrm{T}=85 \%$. In addition, these films are stable under flexing with the sheet resistance varying by $<2 \%$ over more than 1000 bend cycles.

We believe that such films hold great promise as flexible, transparent electrodes. This is especially true if they could be fabricated from low diameter nanowires as this would result in better uniformity and better results for very thin films. As these films can be prepared from the liquid phase we can envisage the preparation of large area electrode by spraying. Such a large area, low cost deposition method is ideal for industry and may be widely implemented should the cost of these materials decrease sufficiently.

\section{Methods}

Silver nanowires were purchased from Seashell Technologies (www.seashelltech.com) as suspensions in isopropyl alcohol $(\mathrm{C}=12.5 \mathrm{mg} / \mathrm{ml}$ as measured by thermogravimetric analysis, Perkin Elmer Pyris). A small volume of the dispersion was diluted down to $0.1 \mathrm{mg} / \mathrm{ml}$ with Millipore water. This was subjected to half an hour low power sonication in a sonic bath (Model Ney Ultrasonic). The 
dispersion was further diluted down to a concentration $\mathrm{C}=0.002 \mathrm{mg} / \mathrm{ml}$ with Millipore water and sonicated for a further 30 minutes.

Silver nanowire films were prepared by vacuum filtration of the above dispersions using porous mixed cellulose ester filter membranes (MF-Millipore Membrane, mixed cellulose esters, Hydrophilic, $0.2 \mu \mathrm{m}, 47 \mathrm{~mm}$ ). The deposited films were transferred to a polyethylene terephthalate (PET) substrate using heat and pressure. ${ }^{25}$ Briefly the PET was placed on a hotplate at $100^{\circ} \mathrm{C}$. The AgNW film / membrane was placed on the PET with the AgNWs in contact with the PET. A $3 \mathrm{~kg}$ weight was then placed on top for two hours. The cellulose filter membrane was then removed by treatment with acetone vapour and subsequent acetone liquid baths followed by a methanol bath. ${ }^{25}$ The film area was $36 \mathrm{~mm}$ in diameter. Thin silver films were prepared using an Edwards S Auto 306 evaporator. Commercially available ITO was purchased from UQG Optics Ltd.

Transmission scans were made using an Epson Perfection V700 Photo flat-bed transmission scanner with a bit depth of 48 bits per pixel and a spatial resolution of 6400 dpi. The numerical output of the scanner was calibrated by scanning a range of neutral density filters. The resultant calibration curve was used to transform the output to represent transmittance. This results in a transmittance map with a transmittance value for every pixel. Transmission maps were transformed into absorbance maps by applying $A=-\log T$ to each pixel. The mean and standard deviation of the transmittance or absorbance were calculated from the entire data set i.e. from the entire set of pixel values. Scanning electron microscopy measurements were made using a Hitachi S-4300 Field Emission scanning electron microscope. Charging was avoided by transferring the nanotube film from cellulose membrane to a glass substrate coated with a thin gold/palladium film. Atomic force microscope images were obtained using a Dimension V AFM. In order to extract the topography and conductance data simultaneously the microscope was operated in the conductance imaging mode (C-AFM). In this technique the AFM tip acts like a mobile probe on the surface and is held at ground potential and a DC bias is applied to the sample. The $\mathrm{z}$ feedback signal is used to generate a normal contact mode AFM topographic profile and the current passing between the tip and the sample is measured using a preamplifier to generate the conductance image. A bias voltage of $0.2 \mathrm{mV}$ up to 1 Volt is applied to the electrode on the surface that drives current through the tubes. A current range of $2 \mathrm{pA}$ to $1 \mu \mathrm{A}$ can be detected by the preamplifier in the CI-AFM module. For this purpose a $\mathrm{Cr} / \mathrm{Pt}$ coated conductive tip with a force constant of $3 \mathrm{~N} / \mathrm{m}$ and a resonant frequency of $75 \mathrm{KHz}$ was employed. In all cases, the loading force employed during measurement was approximately $15 \mathrm{nN}$. (The tips were purchased from Budget Sensors, ElectriMulti 75). Optical transmission spectra were recorded using a Cary Varian 6000i, with a sheet of PET used as the reference. Sheet resistance measurements were made using the four probe technique with silver paste electrodes of dimensions and spacings typically of $\sim \mathrm{mm}$ in size and a Keithley 2400 source meter. Electromechanical measurements were made using a Zwick Z0.5 
Proline tensile tester. The AgNW film on PET was bent into a semicircle which was constrained by the grips of the tensile tester. The film was connected via two electrodes (attached to the grips) to a Keithley KE 2601. The bend radius was then defined by the distance between the grips. The inter-grip distance was then oscillated between typically $15 \mathrm{~mm}$ and $5 \mathrm{~mm}$ over many cycles. LabVIEW software recorded film resistance, inter-grip distance and cycle number.

\section{Acknowledgements}

We acknowledge the Science Foundation Ireland funded collaboration (SFI grant 03/CE3/M406s1) between Trinity College Dublin, University College Cork and Hewlett Packard, Dublin Inkjet Manufacturing Operation which as allowed this work to take place.

\section{Figures}



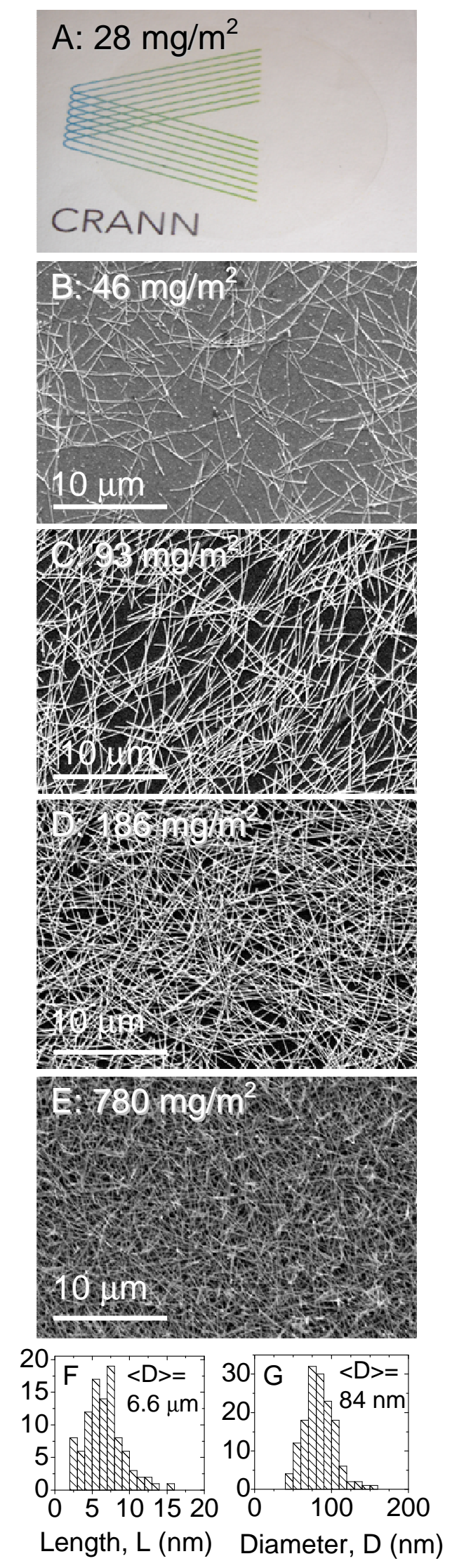

Figure 1: Initial investigation of the films studied in this work. A) Photograph of a film of AgNWs on PET covering the CRANN logo. B-E) SEM images of the surfaces of films of increasing thickness, 
$\left.46 \leq \mathrm{M} / \mathrm{A} \leq 780 \mathrm{mg} / \mathrm{m}^{2} . \mathrm{F}\right)$ and $\left.\mathrm{G}\right)$ Statistics relating to the length $(\mathrm{F})$ and diameter $(\mathrm{G})$ of the individual nanowires.

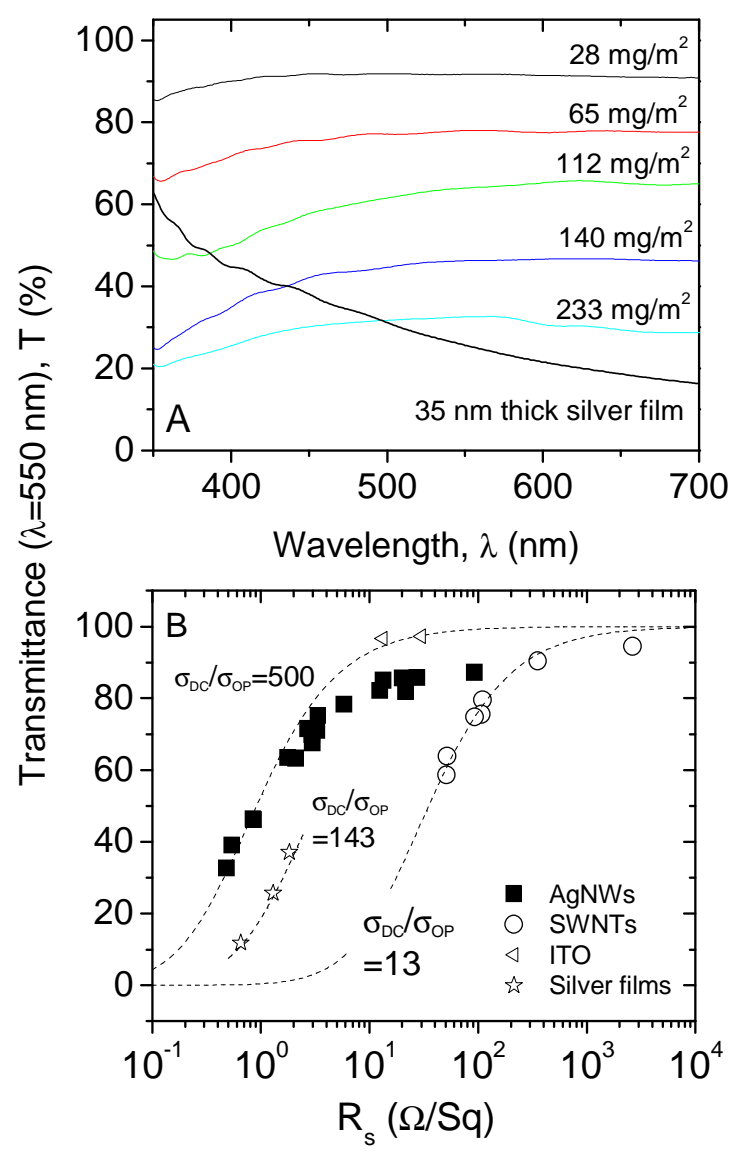

Figure 2: A) Film transmittance spectra for selected AgNW films at a range of thicknesses. Also shown is the spectrum of a $35 \mathrm{~nm}$ thick silver film. B) Transmittance $(\lambda=550 \mathrm{~nm})$ plotted as a function of film sheet resistance for the films studied in this work. The solid symbols represent films of AgNWs. For context, data is also given for films of evaporated silver, commercial ITO and single walled carbon nanotubes. 


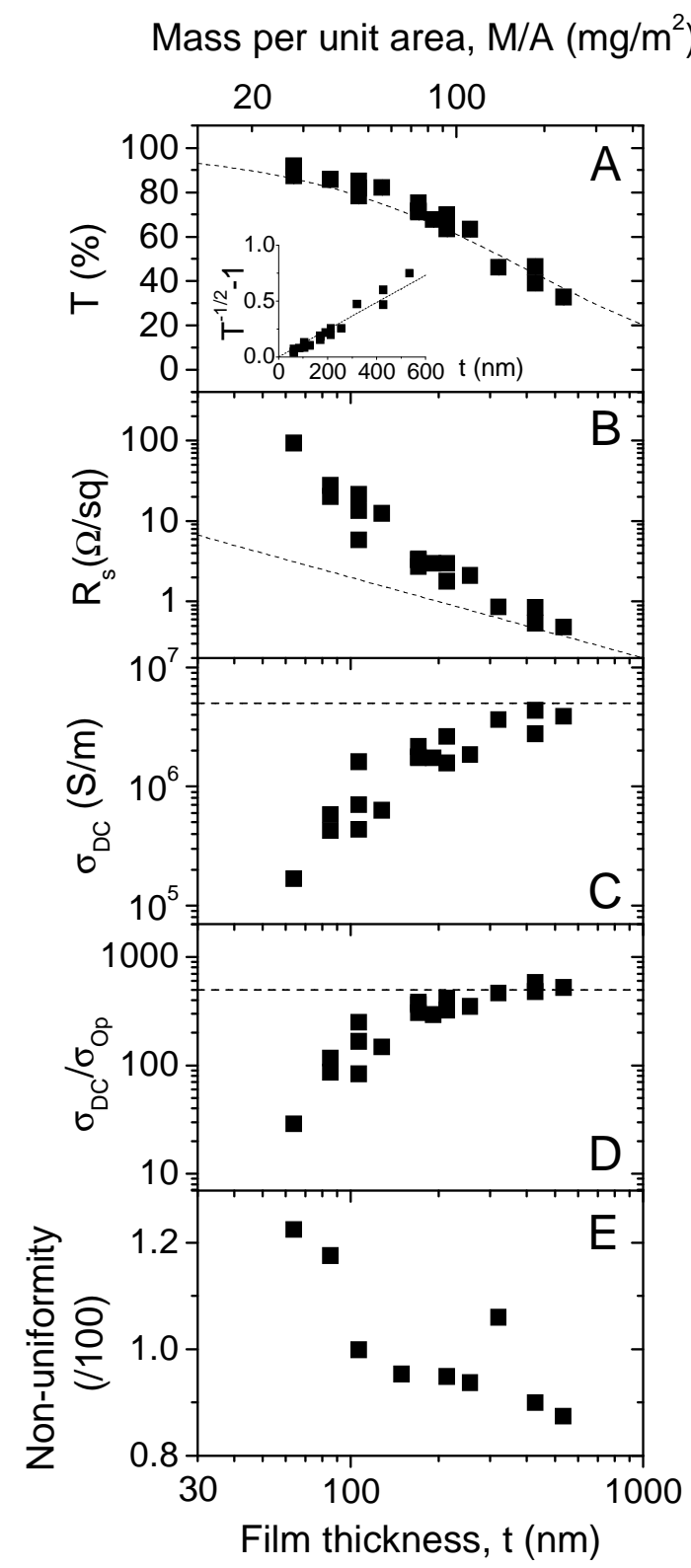

Figure 3: Properties of AgNW films as a function of film thickness, t. For completeness the deposited mass per unit area is shown on the top axis. A) Optical transmittance, measured at $550 \mathrm{~nm}$. The dotted line is a fit to equation 2, consistent with an optical conductivity of $6472 \mathrm{~S} / \mathrm{m}$. B) Sheet resistance. The dotted line represents bulk-like behaviour as given by $R_{s}=1 /\left(\sigma_{D C} t\right)$, where $\sigma_{\mathrm{DC}}=5 \times 10^{6} \mathrm{~S} / \mathrm{m}$ is the DC conductivity. C) DC conductivity, calculated from $R_{s}=1 /\left(\sigma_{D C} t\right)$. The dotted line illustrates a DC conductivity of $5 \times 10^{6} \mathrm{~S} / \mathrm{m}$. D) Ratio of DC to optical conductivity as calculated from the $\mathrm{T}$ and $\mathrm{R}_{\mathrm{s}}$ data. The dotted line illustrates conductivity ratio of 500. E) Local non-uniformity of films, defined as the standard deviation of the local absorbance measured with a pixel sixe of 4 microns. 


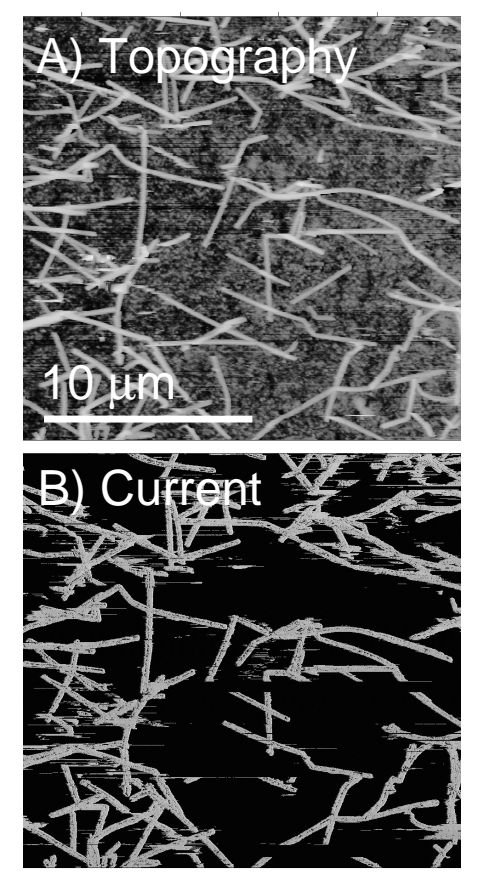

Figure 4: A) Topographic AFM image of a sparse (M/A=10 mg/m²) AgNW film. B) C-AFM current map taken of the same section of film.

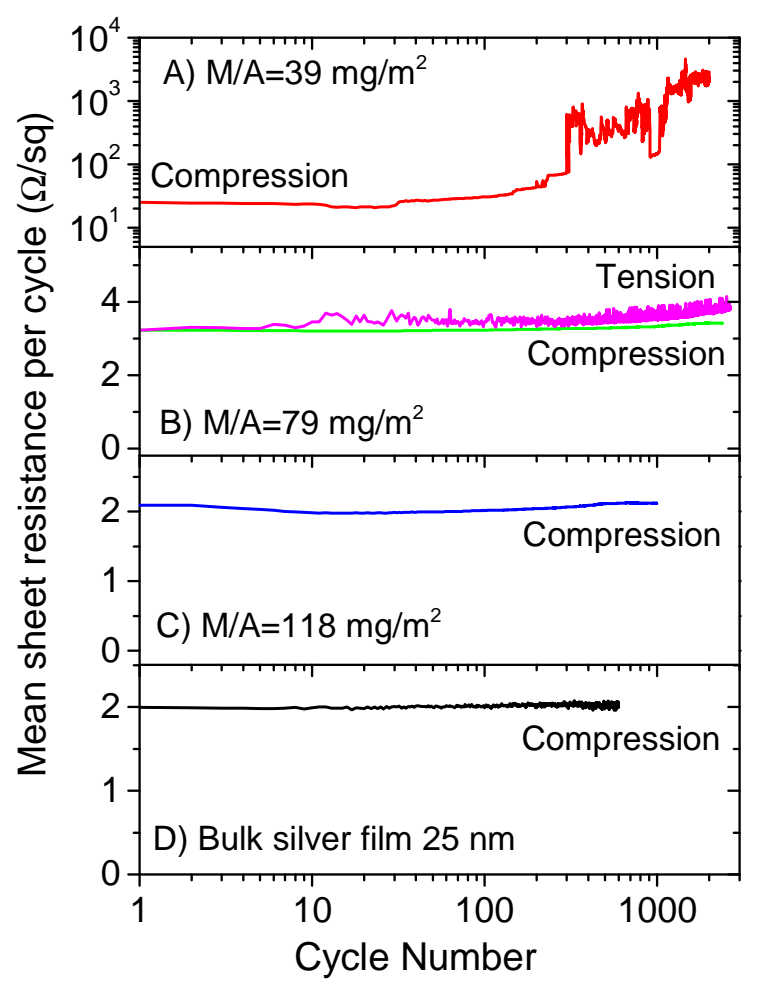

Figure 5: Sheet resistance as a function of cycle number for $\mathrm{AgNW}$ films of three different thicknesses; A) $M / A=39 \mathrm{mg} / \mathrm{m}^{2}$ ( $\left.\mathrm{t}=90 \mathrm{~nm}\right), \mathrm{M} / \mathrm{A}=79 \mathrm{mg} / \mathrm{m}^{2}(\mathrm{t}=182 \mathrm{~nm}), \mathrm{M} / \mathrm{A}=118 \mathrm{mg} / \mathrm{m}^{2}(\mathrm{t}=271 \mathrm{~nm})$. Note all films were measured in compression while the film in B) was also measured in tension. 


\section{References}

1. $\quad$ Bhosle, V.; Prater, J. T.; Yang, F.; Burk, D.; Forrest, S. R.; Narayan, J., Gallium-doped zinc oxide films as transparent electrodes for organic solar cell applications. Journal of Applied Physics 2007, 102, 023501.

2. Yang, F.; Forrest, S. R., Organic solar cells using transparent SnO2-F anodes. Advanced Materials 2006, 18, 2018-2022.

3. Meiss, J.; Riede, M. K.; Leo, K., Towards efficient tin-doped indium oxide (ITO)-free inverted organic solar cells using metal cathodes. Applied Physics Letters 2009, 94, 013303

4. O'Connor, B.; Haughn, C.; An, K. H.; Pipe, K. P.; Shtein, M., Transparent and conductive electrodes based on unpatterned, thin metal films. Applied Physics Letters 2008, 93, 223304.

5. Kang, M. G.; Kim, M. S.; Kim, J. S.; Guo, L. J., Organic Solar Cells Using Nanoimprinted Transparent Metal Electrodes. Advanced Materials 2008, 20, 4408-4413.

6. Tvingstedt, K.; Inganas, O., Electrode grids for ITO-free organic photovoltaic devices. Advanced Materials 2007, 19, 2893-2897.

7. $\quad$ De, S.; Lyons, P. E.; Sorrel, S.; Doherty, E. M.; King, P. J.; Blau, W. J.; Nirmalraj, P. N.; Boland, J. J.; Scardaci, V.; Joimel, J.; Coleman, J. N., Transparent, flexible, and highly conductive thin films based on polymer-nanotube composites ACS Nano 2009, 3, 714-720.

8. $\quad$ Geng, H. Z.; Kim, K. K.; So, K. P.; Lee, Y. S.; Chang, Y.; Lee, Y. H., Effect of acid treatment on carbon nanotube-based flexible transparent conducting films. Journal of the American Chemical Society 2007, $129,7758-7759$.

9. Zhou, Y. H.; Zhang, F. L.; Tvingstedt, K.; Barrau, S.; Li, F. H.; Tian, W. J.; Inganas, O., Investigation on polymer anode design for flexible polymer solar cells. Applied Physics Letters 2008, 92, 233308.

10. Eda, G.; Lin, Y. Y.; Miller, S.; Chen, C. W.; Su, W. F.; Chhowalla, M., Transparent and conducting electrodes for organic electronics from reduced graphene oxide. Applied Physics Letters 2008, 92, 233305.

11. Hong, W. J.; Xu, Y. X.; Lu, G. W.; Li, C.; Shi, G. Q., Transparent graphene/PEDOT-PSS composite films as counter electrodes of dye-sensitized solar cells. Electrochemistry Communications 2008, 10, 15551558 .

12. Kim, K. S.; Zhao, Y.; Jang, H.; Lee, S. Y.; Kim, J. M.; Ahn, J. H.; Kim, P.; Choi, J. Y.; Hong, B. H., Large-scale pattern growth of graphene films for stretchable transparent electrodes. Nature 2009, 457, 706-710.

13. Vollmer, A.; Feng, X. L.; Wang, X.; Zhi, L. J.; Mullen, K.; Koch, N.; Rabe, J. P., Electronic and structural properties of graphene-based transparent and conductive thin film electrodes. Applied Physics aMaterials Science \& Processing 2009, 94, 1-4.

14. Wang, X.; Zhi, L. J.; Mullen, K., Transparent, conductive graphene electrodes for dye-sensitized solar cells. Nano Letters 2008, 8, 323-327.

15. Wu, J. B.; Becerril, H. A.; Bao, Z. N.; Liu, Z. F.; Chen, Y. S.; Peumans, P., Organic solar cells with solution-processed graphene transparent electrodes. Applied Physics Letters 2008, 92, 263302.

16. Artukovic, E.; Kaempgen, M.; Hecht, D. S.; Roth, S.; GrUner, G., Transparent and flexible carbon nanotube transistors. Nano Letters 2005, 5, 757-760.

17. Barnes, T. M.; de Lagemaat, J. V.; Levi, D.; Rumbles, G.; Coutts, T. J.; Weeks, C. L.; Britz, D. A.; Levitsky, I.; Peltola, J.; Glatkowski, P., Optical characterization of highly conductive single-wall carbonnanotube transparent electrodes. Physical Review B 2007, 75, 235410.

18. Contreras, M. A.; Barnes, T.; van de Lagemaat, J.; Rumbles, G.; Coutts, T. J.; Weeks, C.; Glatkowski, P.; Levitsky, I.; Peltola, J.; Britz, D. A., Replacement of transparent conductive oxides by single-wall carbon nanotubes in $\mathrm{Cu}(\mathrm{In}, \mathrm{Ga}) \mathrm{Se}-2$-based solar cells. Journal of Physical Chemistry C 2007, 111, 14045-14048.

19. Fanchini, G.; Unalan, H. E.; Chhowalla, M., Modification of transparent and conducting single wall carbon nanotube thin films via bromine functionalization. Applied Physics Letters 2007, 90, 092114.

20. Geng, H. Z.; Lee, D. S.; Kim, K. K.; Han, G. H.; Park, H. K.; Lee, Y. H., Absorption spectroscopy of surfactant-dispersed carbon nanotube film: Modulation of electronic structures. Chemical Physics Letters 2008, $455,275-278$.

21. Gruner, G., Carbon nanotube films for transparent and plastic electronics. Journal of Materials Chemistry 2006, 16, 3533-3539.

22. Kaempgen, M.; Duesberg, G. S.; Roth, S., Transparent carbon nanotube coatings. Applied Surface Science 2005, 252, 425-429.

23. Ng, M. H. A.; Hartadi, L. T.; Tan, H.; Poa, C. H. P., Efficient coating of transparent and conductive carbon nanotube thin films on plastic substrates. Nanotechnology 2008, 19, 205703. 
24. Parekh, B. B.; Fanchini, G.; Eda, G.; Chhowalla, M., Improved conductivity of transparent single-wall carbon nanotube thin films via stable postdeposition functionalization. Applied Physics Letters 2007, 90, 121913.

25. Wu, Z. C.; Chen, Z. H.; Du, X.; Logan, J. M.; Sippel, J.; Nikolou, M.; Kamaras, K.; Reynolds, J. R.; Tanner, D. B.; Hebard, A. F.; Rinzler, A. G., Transparent, conductive carbon nanotube films. Science 2004, 305, 1273-1276.

26. Aguirre, C. M.; Auvray, S.; Pigeon, S.; Izquierdo, R.; Desjardins, P.; Martel, R., Carbon nanotube sheets as electrodes in organic light-emitting diodes. Applied Physics Letters 2006, 88, 183104

27. Barnes, T. M.; Wu, X.; Zhou, J.; Duda, A.; van de Lagemaat, J.; Coutts, T. J.; Weeks, C. L.; Britz, D. A.; Glatkowski, P., Single-wall carbon nanotube networks as a transparent back contact in CdTe solar cells. Applied Physics Letters 2007, 90, 243503.

28. Lee, J. Y.; Connor, S. T.; Cui, Y.; Peumans, P., Solution-processed metal nanowire mesh transparent electrodes. Nano Letters 2008, 8, 689-692.

29. Dressel, M.; Gruner, G., Electrodynamics of Solids: Optical Properties of Electrons in Matter. Cambridge University Press: Cambridge, 2002.

30. Doherty, E. M.; De, S.; Lyons, L.; Shmelov, A.; Nirmalraj, P. N.; Scardaci, V.; Blau, W. J.; Boland, J. J.; Coleman, J. N., Transparent, conductive films from single walled nanotubes: Film morphology, uniformity and electromechanical stability. Carbon 2009, doi:10.1016/j.carbon.2009.04.040.

31. de Vries, A. J.; Kooij, E. S.; Wormeester, H.; Mewe, A. A.; Poelsema, B., Ellipsometric study of percolation in electroless deposited silver films. Journal of Applied Physics 2007, 101, 053703.

32. Ruzicka, B.; Degiorgi, L.; Gaal, R.; Thien-Nga, L.; Bacsa, R.; Salvetat, J. P.; Forro, L., Optical and dc conductivity study of potassium-doped single-walled carbon nanotube films. Physical Review B 2000, 61, R2468-R2471.

33. Lyons, P. E.; De, S.; Blighe, F.; Nicolosi, V.; Pereira, L. P. C.; Ferreira, M. S.; Coleman, J. N., The relationship between network morphology and conductivity in nanotube films. Journal of Applied Physics 2008, 104, 044302.

34. Stahl, H.; Appenzeller, J.; Lengeler, B.; Martel, R.; Avouris, P., Investigation of the inter-tube coupling in single-wall nanotube ropes. Materials Science and Engineering: C 2001, 15, 291-294.

35. Chen, Z.; Cotterell, B.; Wang, W., The fracture of brittle thin films on compliant substrates in flexible displays. Engineering Fracture Mechanics 2002, 69, 597-603.

36. Leterrier, Y.; Medico, L.; Demarco, F.; Manson, J. A. E.; Betz, U.; Escola, M. F.; Olsson, M. K.; Atamny, F., Mechanical integrity of transparent conductive oxide films for flexible polymer-based displays. Thin Solid Films 2004, 460, 156-166.

37. Shin, D. H.; Shim, H. C.; Song, J.-W.; Kim, S.; H, C.-S., Conductivity of films made from singlewalled carbon nanotubes in terms of bundle diameter. Scripta Materialia 2009, 60, 607-610.

38. Wang, C.; Hu, Y. J.; Lieber, C. M.; Sun, S. H., Ultrathin Au nanowires and their transport properties. Journal of the American Chemical Society 2008, 130, 8902-8903. 